\title{
Testing a New Anticoagulation Method for Free Flap Reconstruction of Head and Neck Cancers
}

\author{
Ebrahim Karimi ${ }^{1}$ Seyyed Hadi Samimi Ardestani ${ }^{1} \cdot$ Mehrdad Jafari $^{2} \cdot$ Ali Bagheri Hagh $^{3}$ \\ ${ }^{1}$ Department of Otolaryngology, Amiralam Hospital, Tehran University of Medical Sciences, Tehran; ${ }^{2}$ Otolaryngology Research Center, Imam \\ Khomeini Hospital, Tehran University of Medical Sciences, Tehran; ${ }^{3}$ Department of Otolaryngology, Baqiyatallah University of Medical Sciences, \\ Tehran, Iran
}

Objectives. Free flaps are widely used to reconstruct head and neck defects. Despite the improvement in the surgical techniques and the surgeons' experiences, flap failures still occur due to thrombotic occlusion after small vessels anastomosis. To reduce the possibility of flap loss as a result of thrombotic occlusion, various anticoagulants have been used. In this study we decided to evaluate a new protocol for anticoagulation therapy and its effect on flap survival and complications.

Methods. In this interventional study, 30 patients with head and neck cancer underwent surgical defects were reconstructed by microvascular free flap between 2013 and 2014. In the postoperative period patients have taken aspirin (100 $\mathrm{mg} /$ day) for 5 days and enoxaparin ( $40 \mathrm{mg} /$ day subcutaneously) for 3 days. The flap survival was followed for three weeks.

Results. Given that there was no complete necrosis or loss of flap, the free flap success rate was as much as $100 \%$. The need for re-exploration occurred in 3 patients $(10 \%)$. Only in one patient the need for re-exploration was due to problem in venous blood flow.

Conclusion. The aspirin-enoxaparin short-term protocol may be a good choice after free flap transfer in reconstruction of head and neck surgical defects.

Keywords. Free Tissue Flaps; Anticoagulants; Head and Neck Neoplasms

\section{INTRODUCTION}

Free flaps are widely used to reconstruct head and neck defects. Microvascular anastomosis technique is the most crucial aspect of free flap transfer in head and neck surgery [1]. By improving the methods and devices of the surgery the success rate of free flap transfer is increased up to $99 \%$ [2].

Despite the improvement in the surgical techniques and the surgeons' experiences, flap failures still occur due to thrombotic

- Received November 17, 2015

Revised January 4, 2016

Accepted January 27, 2016

- Corresponding author: Ali Bagheri Hagh

Department of Otolaryngology, Baqiyatallah University of Medical

Sciences, Mollasadra St., Tehran, Iran

Tel: +98-21-6658-1628, Fax: +98-21-6658-1628

E-mail: mehrdadj82@yahoo.com occlusion after small vessels anastomosis [3,4]. During the first hours and days after free flap transfer, the flap is perfused only via anastomosed microvascular circulation. Endothelialization of anastomosis lines would be complete in 2 weeks after surgery [5]. A total of $5 \%-25 \%$ of flap failures occur due to circulation problems and may necessitate re-exploration [6]. Studies have revealed that microvascular thrombosis mostly occurs within first 2 to 3 days after operation [7]. Therefore, monitoring of free flaps during the first 24 hours is critical and of particular importance in 3 to 5 days after surgery [8]. To reduce the possibility of flap loss as a result of thrombotic occlusion, various anticoagulants including aspirin, heparin, low molecular weight heparin (LMWH), dextran, and prostaglandin E1 have been used $[3,4,9]$.

Studies have shown that using aspirin was effective in preventing occlusion of microvascular anastomosis. In a study administration of aspirin 10 hours before surgery reduced the

Copyright (C) 2016 by Korean Society of Otorhinolaryngology-Head and Neck Surgery.

This is an open-access article distributed under the terms of the Creative Commons Attribution Non-Commercial License (http://creativecommons.org/licenses/by-nc/4.0)

which permits unrestricted non-commercial use, distribution, and reproduction in any medium, provided the original work is properly cited. 
platelet aggregation and prevented anastomosis occlusion [10]. In another study there were no significant difference between using heparin or LMWH in flap survival; however, heparin was associated with higher risk of complications [11]. In a review article the use of aspirin and LMWH subcutaneously, alone or together as an anticoagulant therapy was recommended [12].

Different protocols have been proposed for anticoagulation therapy in free flap transfer. In this study we decided to evaluate a new protocol for anticoagulation therapy and its effect on flap survival and complications.

\section{MATERIALS AND METHODS}

In this interventional study, 30 patients with advanced head and neck cancer underwent extensive surgical resection and the caused defects were reconstructed by microvascular free flap between 2013 and 2014. Briefly, various types of free flaps were used including radial forearm free flaps (RFFF), rectus abdominis flaps (RAF), anterolateral thigh flaps (ALTF), scapular free flaps (SFF), and iliac crest free flap. Because the vascular pedicle in radial and fibular flaps is very susceptible to atherosclerosis, in patients with risk factors of atherosclerosis such as diabetes mellitus, hypertension and hyperlipidemia, and history of previous atherosclerotic vascular diseases, ALTF was used which is believed not to be affected by atherosclerotic changes. RFFF was used in only one of our high risk patients after careful evaluation of its vascular pedicle with Doppler ultrasonography. Moreover, we tried to be more meticulous and use high magnification of operating microscope for anastomosis of the vessels when calcifications or thick intima were present.

In the postoperative period, aspirin (100 mg/day) was administered for 5 days either by orally or via nasogastric tube. In addition, all patients received $40 \mathrm{mg}$ of enoxaparin subcutaneously once daily for 3 days. Heparin and dextran were not used in postoperative period. The donor and recipient site arteries and veins were washed using a $20 \mathrm{Fr}$ catheter during the surgery. Postoperatively, we monitored the perfusion status by tissue color observation every 1 hour during the first day and then more widely apart and pin prick test every day or if any doubt was present regarding the blood flow. Data including free flap failure, venous and arterial thrombosis, and the need for re-exploration

\section{H I G H L I G H T S}

- No single standard exists for anticoagulation after free flap reconstruction of head and cancers.

- We evaluated aspirin $100 \mathrm{mg}$ daily for 5 days + enoxaparin $40 \mathrm{mg}$ a day for 3 days.

- This protocol was relatively safe and effective for this purpose. were recorded in the postoperative period. The free flap failure was considered as flap necrosis in the period of 3 weeks after surgery.

\section{RESULTS}

From 30 patients who participated in the study, 21 of them $(70 \%)$ were male. The mean age of the patients was 45.06 years old (range, 16 to 70 years old). Nine of the patients $(30 \%$ ) had a history of previous chemoradiation.

The surgeries consist of subtotal glossectomy (14 cases), total glossectomy or total resection of the base of the tongue or floor of the mouth (6 cases), segmental resection of mandible and floor of the mouth (2 cases), retromolar trigon resection (1 case), skull base lesions with dural and orbital involvement (3 cases), neopharyngectomy (1 case), skin involvement after recurrence in neck ( 2 cases), and radical maxillectomy with resection of the involved skin (1 case).

The free flaps used in this study include 15 cases of RFFF, 8 RAF, 4 ALTF, and 2 SFF, and one iliac crest free flap.

No complete necrosis or loss of flap was observed during the postoperative period and the rate of free flap success was $100 \%$. Re-exploration was needed in 3 patients $(10 \%)$. Two of them were due to hematoma caused by bleeding of flap muscles fibers. In current study there were not any impairment in arterial blood flow but in one patient impaired venous flow leaded to reexploration of the pedicle. A partial necrosis of ALTF after glossectomy defect reconstruction was encountered which we believe was due to poor shaping and positioning. The issue was resolved with conservative measures such as reform of the sutures.

We had two mortalities in this series none of which happened in the first three weeks of postoperative period. The flaps were viable at the time of death. The causes of death were opium overdose and uncontrolled intracranial pressure following extensive skull base surgery.

\section{DISCUSSION}

Despite the improvement in surgical techniques and surgeons' experiences, free flaps necrosis and loss due to thrombotic occlusions is still an unfortunate event. Different protocols have been proposed for anticoagulation therapy in free flap transfer. In current study we used a combination of $100 \mathrm{mg} /$ day aspirin for 5 days and $40 \mathrm{mg} /$ day enoxaparin injection for 3 days after surgery. Since no flap failure was seen in patients and the rate of free flap transfer success was $100 \%$ with this protocol, the protocol of the present study seems to work well. In the current protocol the number of days and the doses of anticoagulant therapy were lower than previous studies which may be more cost-effective. 
Urken et al. [13] reported that overall success of the free flap transfer was as much as $93.5 \%$. As the experience of surgeons increased, the complications decreased. Approximately $75 \%$ of the reconstruction carried out in that study was for defects of the oral cavity [13]. In another study by the same author on bone reconstruction by scapular fasciocutaneous flap, the success rate was reported $89 \%$ over 15 years and the flaps failed in 5 patients [14]. Hidalgo and Jones [15] demonstrated the role of immediate exploration on transplant vascular problems in free flap transfer. Eleven of 150 transferred flaps had blood circulation problem during the first hour to 6 days after operation which in 8 cases it was due to venous thrombosis and in 3 of them due to arterial thrombosis. The average time of exploration was 1.5 hours after facing with problem which resulted in rescue of all 11 flaps [15]. In another study by Hidalgo et al. [16] on 716 cases of free flap transfer for oncologic defects, the rate of success was $98 \%$. Eight percent of patients needed re-exploration due to anastomosis complications and infection which rescued 40 of these flaps. In a study by Haughey et al. [17] on 241 patients with free flap transfer the success rate was $95 \%$. Administration of more than $7 \mathrm{~L}$ of crystalloid fluids during surgery and age over 55 years were associated with medical complications. Kessler et al. [18] believed that by improving the quality of patient management, surgical techniques, and careful selection of patients it could be possible to reach a $100 \%$ success rate. From 81 patients with mouth and mandible reconstruction, they only had 4 complications related to anastomosis in which quick action prevented from any flap failures.

Aside from the importance of surgical technique, one of the factors that seem to affect the success rate of free flap is anticoagulant medications before, during or after surgery. There is no unified standard protocol for using anticoagulant medications. In a review by Jokuszies et al. [19] most of the microvascular surgeons used a combination of aspirin and heparin or LMWH. Salgado et al. [12] suggested aspirin and LMWH alone or a combination of them as anticoagulant medications. Dassonville et al. [20] used LMWH as the anticoagulant agent and the result was $6.6 \%$ flap failure with $15 \%$ of cases being re-explored. In two studies by Nakamizo et al. [9] and Fukuiwa et al. [21], prostaglandin E1 was used for 5 days after the operation and the failure rate were $2.7 \%$ and $5.9 \%$, respectively.

Chien et al. [22] used a combination of aspirin ( $325 \mathrm{mg} /$ day) and heparin (5,000 unit subcutaneous per day) which resulted in a success rate of $97.2 \%$. Two-point-eight percent of the cases faced venous congestion which needed further exploration and $5.6 \%$ faced hematoma which needed surgical intervention. The failure rate in Suh et al. [23] study was $0.8 \%$ with $1.8 \%$ rate of re-exploration. Irrigating the donor and recipient veins with heparinized saline solution before operation and $81 \mathrm{mg} /$ day aspirin for 7 days after surgery were effective in reduction of flap failure.

The main limitations of this study are small sample size, and lack of a control group. As the use of free flaps is rapidly growing all around the world, conducting new studies with larger sample sizes in well-designed randomized controlled trials are needed more than ever. However this study, as same as similar previous studies, may be used for data pooling for meta-analyses.

\section{CONFLICT OF INTEREST}

No potential conflict of interest relevant to this article was reported.

\section{REFERENCES}

1. Kim H, Jeong WJ, Ahn SH. Results of free flap reconstruction after ablative surgery in the head and neck. Clin Exp Otorhinolaryngol. 2015 Jun;8(2):167-73.

2. Kruse AL, Luebbers HT, Gratz KW, Obwegeser JA. Factors influencing survival of free-flap in reconstruction for cancer of the head and neck: a literature review. Microsurgery. 2010;30(3):242-8.

3. Froemel D, Fitzsimons SJ, Frank J, Sauerbier M, Meurer A, Barker JH.A review of thrombosis and antithrombotic therapy in microvascular surgery. Eur Surg Res. 2013;50(1):32-43.

4. Stephan B, Schenk JF, Nemeh A, Pindur G. The use of antithrombotic agents in microvascular surgery. Clin Hemorheol Microcirc. 2009;43(1-2):51-6.

5. Rao VK, Nightingale G, O'Brien BM. Scanning electron microscope study of microvenous grafts to artery. Plast Reconstr Surg. 1983 Jan;71(1):98-106.

6. Gilbert RW, Ragnarsson R, Berggren A, Ostrup L. Microvenous grafts to arterial defects: the use of mechanical or suture anastomoses. Arch Otolaryngol Head Neck Surg. 1989 Aug;115(8):970-6.

7. Kroll SS, Schusterman MA, Reece GP, Miller MJ, Evans GR, Robb GL, et al.Timing of pedicle thrombosis and flap loss after free-tissue transfer. Plast Reconstr Surg. 1996 Dec;98(7):1230-3.

8. Hirigoyen MB, Urken ML, Weinberg H. Free flap monitoring: a review of current practice. Microsurgery. 1995;16(11):723-6.

9. Nakamizo M, Yokoshima K, Yagi T. Use of free flaps for reconstruction in head and neck surgery: a retrospective study of 182 cases. Auris Nasus Larynx. 2004 Sep;31(3):269-73.

10. Salemark L, Wieslander JB, Dougan P, Arnljots B. Effect of low and ultra low oral doses of acetylsalicylic acid in microvascular surgery: an experimental study in the rabbit. Scand J Plast Reconstr Surg Hand Surg. 1991;25(3):203-11.

11. Ashjian P, Chen CM, Pusic A, Disa JJ, Cordeiro PG, Mehrara BJ.The effect of postoperative anticoagulation on microvascular thrombosis. Ann Plast Surg. 2007 Jul;59(1):36-9.

12. Salgado CJ, Chim H, Schoenoff S, Mardini S. Postoperative care and monitoring of the reconstructed head and neck patient. Semin Plast Surg. 2010 Aug;24(3):281-7.

13. Urken ML, Weinberg H, Buchbinder D, Moscoso JF, Lawson W, Catalano PJ, et al. Microvascular free flaps in head and neck reconstruction: report of 200 cases and review of complications. Arch Otolaryngol Head Neck Surg. 1994 Jun;120(6):633-40.

14. Urken ML, Bridger AG, Zur KB, Genden EM. The scapular osteofasciocutaneous flap: a 12-year experience. Arch Otolaryngol Head Neck Surg. 2001 Jul;127(7):862-9.

15. Hidalgo DA, Jones CS. The role of emergent exploration in free-tissue transfer: a review of 150 consecutive cases. Plast Reconstr Surg. 1990 Sep;86(3):492-8. 
16. Hidalgo DA, Disa JJ, Cordeiro PG, Hu QY.A review of 716 consecutive free flaps for oncologic surgical defects: refinement in donor-site selection and technique. Plast Reconstr Surg. 1998 Sep;102(3):72232.

17. Haughey BH,Wilson E, Kluwe L, Piccirillo J, Fredrickson J, Sessions D, et al. Free flap reconstruction of the head and neck: analysis of 241 cases. Otolaryngol Head Neck Surg. 2001 Jul;125(1):10-7.

18. Kessler P, Poort L, Bockmann R, Lethaus B. Definition of quality indicators in microsurgery in head and neck reconstruction based on a 5-year follow-up without a loss. J Craniomaxillofac Surg. 2013 Jan; 41(1):2-6.

19. Jokuszies A, Herold C, Niederbichler AD, Vogt PM. Anticoagulative strategies in reconstructive surgery: clinical significance and applicability. Ger Med Sci. 2012;10:Doc01.
20. Dassonville O, Poissonnet G, Chamorey E, Vallicioni J, Demard F, Santini J, et al. Head and neck reconstruction with free flaps: a report on 213 cases. Eur Arch Otorhinolaryngol. 2008 Jan;265(1):8595.

21. Fukuiwa T, Nishimoto K, Hayashi T, Kurono Y. Venous thrombosis after microvascular free-tissue transfer in head and neck cancer reconstruction. Auris Nasus Larynx. 2008 Sep;35(3):390-6.

22. Chien W,Varvares MA, Hadlock T, Cheney M, Deschler DG. Effects of aspirin and low-dose heparin in head and neck reconstruction using microvascular free flaps. Laryngoscope. 2005 Jun;115(6):973-6.

23. Suh JD, Sercarz JA, Abemayor E, Calcaterra TC, Rawnsley JD, Alam $\mathrm{D}$, et al. Analysis of outcome and complications in 400 cases of microvascular head and neck reconstruction. Arch Otolaryngol Head Neck Surg. 2004 Aug;130(8):962-6. 